\title{
Seeing double
}

\author{
Research projects are often developed by multiple groups worldwide, either in collaboration or in a form of \\ genuine competition. In this Editorial, we discuss our approach to dealing with situations where multiple related \\ manuscripts are submitted or published, in our journal or elsewhere, within a short period of time.
}

$\mathrm{t}$ can be a researcher's worst nightmare. During the weekly literature review, you come across a title that sounds ominously similar to the project you have been working on for the past year. Panic. Open the paper and read it in excruciating detail. Partial relief. There are some clear differences between the pieces. But still, there is a significant conceptual overlap. Will this paper stop your work getting published in the journal you wanted? Will it stop you getting published at all? The questions start to swirl. A bad day that most researchers will experience at some point during their career.

The dreaded scoop always seems to come out of left field - at least when you are on the receiving end of it. Yet, with the world population approaching eight billion ${ }^{1}$, the scientific community is growing alike, increasing the likelihood of somebody else having the same good idea that you had. And that's what it really is - affirmation that you are working on a topic that others are interested in. Maybe you even directly or indirectly influenced the generation of this other work through a previous publication or a comment made during a conference presentation. Science is, after all, a collective enterprise. The cross-breeding of ideas is both effective and expected, rather than some mysterious form of synchronicity ${ }^{2}$.

Every journal will take a somewhat different approach to handling the issue of scooping, depending on their target audiences and publishing model. For example, our sister journal, Nature Communications, recently introduced a new anti-scooping policy that recognises the positive influence multiple studies can have on the reinforcement of scientific discovery ${ }^{3}$. At Nature Catalysis we follow similar practices, for instance, we do not consider any competing works that are published while a manuscript is under review in our journal. While a previously published work will factor into our assessment of the potential impact of a current submission, novelty is only one consideration among many. Indeed, claims to be the first to report something are very difficult to prove, which is why it has been longstanding policy at all Nature journals to ask authors to remove language such as 'novel' and 'first time'. Such statements can detract from the achievements of the work by generating discussions about its newness instead of its unique scientific aspects. Interesting, cutting-edge and high-quality manuscripts will stand on their own regardless of competing studies.

A curious case to consider is when two papers reporting similar findings are submitted simultaneously or during an overlapping period of peer review. Well surely this almost never happens? The eagle-eyed amongst you might have noticed two articles with remarkably similar titles on the contents page this month. And no, you are not seeing double. This issue of Nature Catalysis really does contain two papers reporting the electrochemical semi-hydrogenation of acetylene to ethylene on copper-based catalysts. In fact, this is not the first time that highly similar studies have been submitted to the journal simultaneously, though it is the first time that both have ended up being published. Some might wonder why we didn't prioritize one work over the other. As a matter of fairness, we run under the general principle that no unpublished paper (including works under review) will be considered as a prior art when making an initial editorial decision. Take the more common situation where a competing study is under review (and therefore unpublished) at a different journal. We would have no knowledge of the existence of this hypothetical piece and hence it would have no impact on the assessment of novelty at our journal. The same principle should apply in the case of a dual submission to the same journal.

\section{"'As a matter of fairness, we run under the general principle that no unpublished paper (including works under review) will be considered as \\ a prior art when making an initial editorial decision."'"}

The works in question, by Haotian Wang, Tierui Zhang and co-workers and
Xinliang Feng, Jian Zhang and colleagues were submitted to our journal within weeks of each other, and while they share notable similarities, they also possess distinct and complementary features. The main asset of both studies is the use of a gas-diffusion electrode, which allows them to overcome the low solubility of acetylene in aqueous media. Each work developed a copper catalyst - from a $\mathrm{Cu}-\mathrm{Al}$ layered double hydroxide (Shi et al.) and from copper dendrites electrodeposited in situ (Bu et al.) - and tested it in a flow cell setup using a continuous flow of acetylene, achieving large current densities and Faradaic efficiencies for ethylene at mild potentials. The practical implementation of the strategy has been demonstrated using a gas mixture that simulates the ethylene-rich stream produced by steam cracking (containing $0.5-1 \%$ acetylene), continuously producing polymer-grade ethylene with an acetylene content below 10 $\mathrm{ppm}$. The two pieces have been highlighted and discussed together in a combined News and Views article, which appears in the same issue.

As the global quest to reduce carbon emissions and the explosion of green energy production continue apace, it is unsurprising that attempts are made to electrify prominent processes in the chemical industry. The electrosynthesis of ammonia and commodity chemicals, for example, represent rapidly growing fields of research at present. This competition inevitably results in independent works developing simultaneously around different parts of the globe, and in some cases being submitted to the same journal, as in the present case. As discussed above, the two submissions were initially treated independently, and we ensured at every step that consistent decisions were being made along the entire editorial and peer review processes. In such cases, we typically try to ensure that at least one reviewer can comment on both works and that critical issues that apply to both studies are addressed in both of them. Fairness and consistency are always among our main priorities, and for such cases they become even more vital.

Two or more groups simultaneously submitting to the same journal may 
ultimately be a stroke of serendipity. In the more likely event that the timing does not align like this, do not be disheartened. Studies have shown that when it comes to science, coming second does not mean losing the race ${ }^{4}$.

Published online: 22 July 2021

https://doi.org/10.1038/s41929-021-00662-6
References

$\square$ 1. https://www.worldometers.info/world-population/

2. Jung, C. G. Synchronicity: An Acausal Connecting Principle (Princeton Univ. Press, 1973).

3. Nat. Commun. 11, 4466 (2020).

4. Callaway, E. Nature 575, 576-577 (2019). 